\title{
Craniosynostosis-Dandy-Walker malformation-hydrocephalus syndrome
}

INSERM

\section{Source}

INSERM. (1999). Orphanet: an online rare disease and orphan drug data base.

Craniosynostosis-Dandy-Walker malformation-hydrocephalus syndrome. ORPHA:1538

Craniosynostosis, Dandy-Walker malformation and hydrocephalus is a malformation disorder characterized by sagittal craniosynostosis (see this term), Dandy-Walker malformation, hydrocephalus, craniofacial dysmorphism (including dolichocephaly, hypertelorism, micrognathia, positional ear deformity) and variable developmental delay. The inheritance pattern appears to be autosomal dominant. 